\title{
Cognitive impairment, clinical symptoms and functional disability in patients emerging from the minimally conscious state
}

\author{
Yelena G. Bodien ${ }^{\mathrm{a}, \mathrm{b}, *}$, Geraldine Martens ${ }^{\mathrm{a}, \mathrm{c}}$, Joseph Ostrow ${ }^{\mathrm{a}}$, Kristen Sheau ${ }^{\mathrm{a}}$ and Joseph T. Giacino ${ }^{\mathrm{a}}$ \\ ${ }^{a}$ Department of Physical Medicine and Rehabilitation, Spaulding Rehabilitation Hospital, Harvard Medical \\ School, Boston, MA, USA \\ ${ }^{\mathrm{b}}$ Department of Neurology, Massachusetts General Hospital, Harvard Medical School, Boston, MA, USA \\ ${ }^{\mathrm{c}}$ Department of Neurology and GIGA-Consciousness, Coma Science Group, GIGA Research, University and \\ University Hospital of Liege, Liege, Belgium
}

\begin{abstract}
.
BACKGROUND: Although emergence from the minimally conscious state (eMCS) is associated with symptoms including disorientation, memory and attention impairment, restlessness, and significant functional disability, the neurobehavioral profile of eMCS has not been empirically characterized.

OBJECTIVE: Determine degree of cognitive impairment, presence of clinical symptoms and functional disability at time eMCS in patients with traumatic and non-traumatic brain injury (TBI, nTBI).

METHODS: Retrospective observational study of 169 adults (median [interquartile range] age: 51 [29, 62] years; male: 116; TBI: 103) who emerged from MCS based on the Coma Recovery Scale-Revised while in an inpatient Disorders of Consciousness program. Outcome measures include the Confusion Assessment Protocol (CAP) and Disability Rating Scale (DRS).

RESULTS: CAP administration was attempted in 54 subjects. Twenty-eight subjects had valid scores on all CAP items, with a median [interquartile range] of 4 [3-5] symptoms of confusion. Scores in $93 \%$ of this subsample were consistent with an acute confusional state. The most common symptoms were cognitive impairment (98\% of subjects), disorientation (93\%), and agitation (69\%). The median DRS score upon emergence from MCS was $14.5[13,16]$, indicating severe disability $(n=140)$.

CONCLUSIONS: eMCS is associated with an acute confusional state and severe disability. This finding may inform the lower boundary of confusion as well as approach to treatment and caregiver education.
\end{abstract}

Keywords: Brain injury, traumatic brain injury, consciousness, confusion

\footnotetext{
*Address for correspondence: Yelena G. Bodien, Department of Physical Medicine and Rehabilitation, 300 First Ave, Charlestown, Spaulding Rehabilitation Hospital, MA 02129, USA. Tel.: +1 617952 6308; E-mail: ybodien@mgh.harvard.edu.
}

\section{Abbreviations}
ABS
ACS
Agitated Behavioral Scale
CAP acute confusional state
CRS-R 


$\begin{array}{ll}\text { DRS } & \text { Disability Rating Scale } \\ \text { DoC } & \text { disorders of consciousness } \\ \text { eMCS } & \text { emerged from minimally conscious state } \\ \text { GOAT } & \text { Galveston Orientation and Amnesia Test } \\ \text { IQR } & \text { interquartile range } \\ \text { ME } & \text { median } \\ \text { MCS } & \text { minimally conscious state } \\ \text { nTBI } & \text { non-traumatic brain injury } \\ \text { PTA } & \text { post-traumatic amnesia } \\ \text { PTCS } & \text { post-traumatic confusional state } \\ \text { TBI } & \text { traumatic brain injury } \\ \text { VS } & \text { vegetative state }\end{array}$

\section{Introduction}

Severe brain injury may lead to prolonged impairments in arousal and awareness, key components of human consciousness. Broadly referred to as disorders of consciousness (DoC), the stages of recovery from brain-injury-induced coma are: vegetative state (VS, spontaneous eye-opening but no overt signs of awareness), (Giacino, 2004) minimally conscious state (MCS, unequivocal but intermittent evidence of awareness) (Giacino et al., 2002) and emergence from MCS (eMCS, recovery of functional object use or communication). (Giacino et al., 2002) eMCS patients are typically disoriented, cognitively impaired, (Nakase-Richardson, Yablon, Sherer, Evans, \& Nick, 2008; Nakase-Richardson, Yablon, Sherer, Nick, \& Evans, 2009) inattentive, (Stuss et al., 1999) and unaware of the circumstances surrounding their injury and level of disability. Clinical symptoms accompanying this phase of recovery include sleep disruptions, poor daytime arousal, and perceptual disturbance (Nakase-Thompson, Sherer, Yablon, Nick, \& Trzepacz, 2004). Fluctuation across and within these symptom domains creates challenges for assessment, treatment, and prognostication of patients recovering from severe brain injury. Patients emerging from MCS remain largely dependent for daily activities and frequently require constant supervision to ensure safety (Nakase-Richardson et al., 2009). This phase of recovery can be especially difficult for families enthusiastic about the return of communication but surprised by significant, lingering impairments in cognition and behavioral regulation. Despite widespread clinical descriptions of the transition from MCS into a confusional state, the extended period of recovery that follows eMCS and precedes stabilization of cognition and behavioral regulation is not systematically defined in the literature.

The cognitive and behavioral profile associated with eMCS patients likely coincides with that of a confusional state. In fact, the post-traumatic confusional state (PTCS), (Sherer, Nakase-Thompson, Yablon, \& Gontkovsky, 2005) a recently adopted diagnostic term which specifically requires assessment of the collection of symptoms most common among severe traumatic brain injury (TBI) patients, is considered to be on the DoC spectrum (Giacino, Fins, Laureys, \& Schiff, 2014). PTCS is characterized by cognitive impairment, disorientation, agitation/restlessness, disturbances in sleep and arousal, and psychotic-type features (e.g., delusions and perceptual disturbances) which may fluctuate in severity over the course of minutes to hours (Nakase-Richardson et al., 2009; Nakase-Thompson et al., 2004). Thus PTCS is a clinically important sub-epoch of the period from eMCS to cognitive and behavioral recovery that is consistent with return of basic functional independence. A growing body of literature focused on describing PTCS with a standardized assessment tool, the Confusion Assessment Protocol (CAP), (Sherer et al., 2005) has allowed clinicians and researchers to reliably and comprehensively assess this phase of recovery. Several studies have identified the confusional state as a prognostic indicator for poor cooperation during rehabilitation (Silva et al., 2012) and decreased productivity and employment at 1 year after TBI (Nakase-Richardson, Yablon, \& Sherer, 2007; Sherer, Yablon, NakaseRichardson, \& Nick, 2008; Sherer, Yablon, \& Nick, 2013).

Despite increased focus on the characterization of MCS and PTCS, the transition between MCS and the confusional state is poorly understood. At least one study has found that, although eMCS is associated with increased responsiveness, accuracy for basic orientation questions remains impaired (Nakase-Richardson et al., 2009). However, it remains unclear whether eMCS and PTCS are distinct or overlapping phases of recovery. This gap in knowledge is especially problematic for caregivers who may not know what to expect when basic communication has returned but ongoing, marked confusion persists. Furthermore, a thorough understanding of the symptoms that accompany transition out of MCS will improve clinicians' ability to plan treatment, anticipate fluctuations that are a natural part of recovery, evaluate the effectiveness of interventions, and predict outcome. 
In this retrospective database study, we address the following aims: 1) determine whether eMCS patients demonstrate the cognitive impairments and behavioral dysregulation consistent with a confusional state, 2) describe the level of functional disability of eMCS patients, and 3) evaluate whether etiology (i.e., traumatic versus non-traumatic) influences the cognitive and functional profile of eMCS patients. For clarity, we use the term acute confusional state (ACS) rather than PTCS to highlight the etiologically heterogeneous nature (i.e., TBI and nTBI) of our sample.

\section{Material and methods}

The study was approved by the local institutional review board. The original raw data are available on request.

\subsection{Participants}

Demographic and behavioral data were retrospectively obtained from a REDCap (Harris et al., 2009) database that curates clinical metrics collected in a specialized DoC program housed at a long term acute care hospital and an inpatient rehabilitation facility. The admission requirements to the DoC program and the DoC program structure are the same at both facilities. Inclusion criteria were as follows: (1) acquired brain injury with a Coma Recovery Scale-Revised (CRS-R) (Giacino, Kalmar, \& Whyte, 2004) diagnosis of VS or MCS on admission to rehabilitation; (2) at least 17 years old; and (3) emerged from MCS during inpatient rehabilitation based on the CRS-R. Patients with evidence of functional disability prior to brain injury were excluded. eMCS was defined based on observation of functional object use and/or functional communication on two consecutive examinations. Patients were determined to have primarily a TBI or nTBI (e.g., anoxic-ischemic injury, infection) based on medical chart review. When both TBI and nTBI etiologies were recorded in the chart $(n=4)$, a medical chart review was conducted by YGB to determine the etiology that most likely contributed to the DoC.

\subsection{Description of methods}

Evidence of functional object use or functional communication on the CRS-R, a standardized and validated 23 -item hierarchical scale that assesses auditory, visual, motor, and oromotor function as well as communication ability and arousal in patients with impaired consciousness, was used as the diagnostic criteria for eMCS. The following outcome measures were collected: (1) time to emergence from MCS (i.e., number of days from injury to the first of two consecutive assessments indicating eMCS on the CRS-R); (2) CAP: number and domain of confusion symptoms, cognitive impairment subscale score $(\mathrm{CI}$, range $=0-28,<18$ indicates confusion), and Galveston Orientation and Amnesia Test total score (GOAT, range $=-8-100,<75$ indicates confusion) (Levin, O'Donnell, \& Grossman, 1979) score within seven days of emergence from MCS; and (3) Disability Rating Scale (DRS) (Rappaport, Hall, Hopkins, Belleza, \& Cope, 1982) total score within three days of emergence from MCS. The CAP is a seven-item composite measure that assesses cognition, disorientation, restlessness, symptom fluctuation, sleep/wake cycle disruption, and psychotic-type symptoms. The CAP includes items from the TOTART, (Stuss et al., 1999) GOAT, Agitated Behavioral Scale (ABS) (Bogner, Corrigan, Stange, \& Rabold, 1999) and Delirium Rating Scale-Revised-98 (Trzepacz et al., 2001). Performance on cognitive measures, the GOAT, and clinical behavioral items contribute to a total confusional score ranging from 0-7 symptoms. Four or more symptoms, or three or more symptoms if one of the symptoms is disorientation, indicate PTCS. The $\mathrm{CAP}$ was designed for patients with confusional state caused by TBI, however, it is also used to assess patients with nTBI because no comparable measures are available for this population. We chose the CAP score occurring within 7 days of eMCS diagnosis because weekly standardized assessment of confusion, once criteria for eMCS are met, is a core component of the DoC inpatient rehabilitation program. The DRS is a measure of functional disability designed to assess patients who have sustained a brain injury [16]. The DRS total score ranges from 0 (no disability) to 29 (extreme vegetative state). We chose the DRS score occurring within 3 days of eMCS diagnosis because the DRS was assessed biweekly in most patients and is intended to reflect an observation period of 72 hours. The CRS-R, CAP Cognitive Impairment and GOAT items were administered by trained speech language pathologists and occupational therapists at least weekly. The CAP clinical-observed items were scored by nursing staff, with input from speech language pathologists and occupational and physical therapists. The DRS was 
scored by the rehabilitation team at biweekly, interdisciplinary team meetings.

\subsection{Statistical analyses}

We measured between-group demographic differences using non-parametric tests (Mann-Whitney U Test, McNemars Test) and time to eMCS with logistic regression accounting for age. All analyses used the STATA statistical software (version 14.2) (StataCorp, 2015).

\section{Results}

We obtained data from 172 patients (days post injury median $(\mathrm{Me})$ interquartile range [IQR]: 50 [42-75], range:18-325) collected between 2012 and 2017. We subsequently excluded three patients because the number of days from injury to eMCS exceeded the upper boundary of the third quartile, leaving 169 subjects (Table 1, Fig. 1) in the analysis with the following characteristics: 116 males, Me [IQR] 51 [29, 62] years old on admission, 50 [42, 71] days from injury to eMCS, and 103 with TBI. Patients with TBI were significantly younger compared with patients with nTBI (TBI: 44 [24, 58] years, nTBI: 58.5 [45, 70] years, Mann-Whitney U Test $p<0.0001$ ) and there was a significantly higher proportion of males in the TBI group $(\mathrm{TBI}=77.7 \%, \mathrm{nTBI}=54.6 \%$, McNamers Test $p<0.0001)$. Median regression analysis of days from injury to eMCS including age and sex as a co-variates revealed a significant difference between etiologies (TBI: 49 [40, 67] days, nTBI: 56 $[44,80]$ days, $p=0.03,95 \%$ confidence interval $[\mathrm{CI}]$ [-19.02-0.98]).

The CAP was attempted within seven days of eMCS diagnosis in $35 \%(n=54)$ of the sample. Factors contributing to missing CAP data in the remaining $65 \%$ of the sample could not be determined. However, the group with and without CAP data did not differ in etiology (proportion of TBI versus non-TBI patients), days from injury to eMCS, or DRS scores at emergence from eMCS (all $p$ values $>0.05$ ). Of these 54 subjects, complete, valid, CAP scores were available in 52\% $(n=28)$ and were obtained $4[1,6]$ days after eMCS. In the remaining $48 \%$, scores were either confounded by aphasia, impairments in phonation and visual-perceptual dysfunction or were missing for undocumented reasons (Fig. 1). At time of eMCS, the Me [IQR] number of symptoms of confusion was 4 [3-5] and scores in $93 \%(n=27)$ of the sample were consistent with an ACS (Table 2, Fig. 2). The most common symptoms of confusion, which were based on the number of subjects who had a valid score for each symptom assessment, were cognitive impairment (98\%, 39 of 40 subjects), disorientation ( $93 \%, 38$ out of 41 subjects), agitation (69\%, 31 out of 45 subjects), and symptom fluctuation (44\%, 20 out of 45 subjects) (Fig. 3). The Me [IQR] cognitive impairment score was 8 and GOAT total score was 32. There were no significant differences between number or category of symptoms of confusion between patients with TBI versus nTBI.

We assessed global function at emergence from MCS in subjects with valid DRS scores recorded within three days of emergence from MCS ( $n=140$, $83 \%$ ). Twenty-nine subjects were missing DRS data due to factors that were not documented. Median [IQR] days between eMCS and DRS assessment was 2 [1,3]. Total scores ranged from 5 (moderate disability) to 25 (vegetative state), suggesting a wide spread in level of function at emergence from MCS. The Me [IQR] DRS score of 14.5 was in the severe disability category (Fig. 4). There were no significant differences in median DRS score between patients with TBI versus nTBI (Table 2).

\section{Discussion}

Recovery from severe brain injury generally evolves over a continuum, beginning with a period of coma that progresses to VS, then MCS followed by eMCS. We found that nearly all eMCS patients

Table 1

Patient characteristics

\begin{tabular}{lcccc}
\hline & All & TBI & nTBI & TBI vs nTBI \\
& $n=169$ & $n=103$ & $n=66$ & $p$-value \\
\hline Age, years median [IQR] & $51[29,62]$ & $44[24,58]$ & $58.5[45,70]$ & $<0.0001^{\mathrm{a}}$ \\
Sex, male n (\%) & $116(68.6)$ & $80(77.7)$ & $36(54.6)$ & $<0.0001^{\mathrm{b}}$ \\
Time from injury to eMCS, days median [IQR] & $50[42,71]$ & $49[40,67]$ & $56[44,80]$ & $0.03^{\mathrm{c}}$ \\
\hline
\end{tabular}

${ }^{\mathrm{a}}$ Mann-Whitney U Test, ${ }^{\mathrm{b}} \mathrm{McNemars}$ Test, ${ }^{\mathrm{c}}$ Median regression with age and sex as co-variates. 


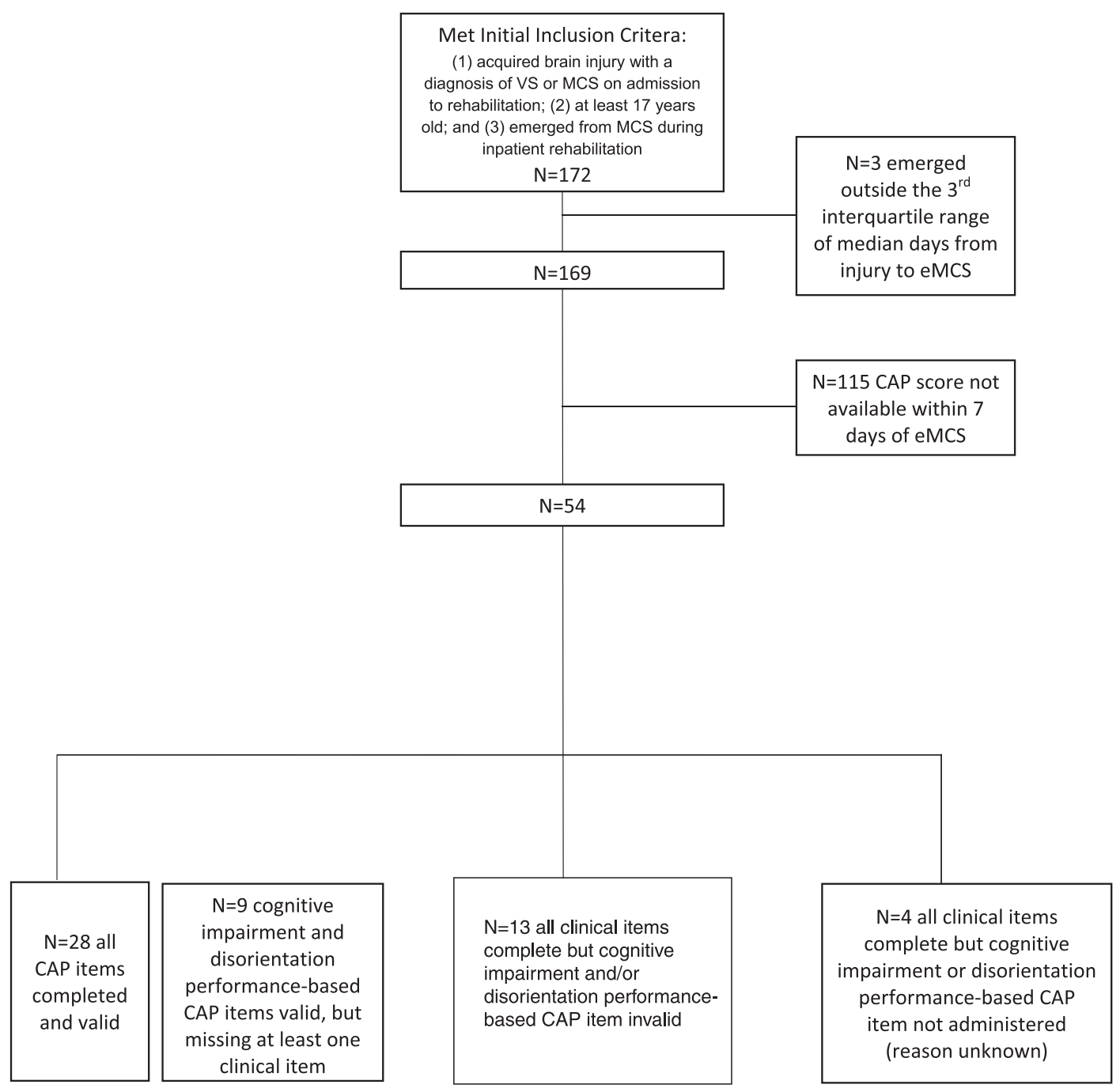

Fig. 1. Subject inclusion flow-chart for analysis of Confusion Assessment Protocol data.

Table 2

Cognition, disorientation and disability at time of emergence from MCS

\begin{tabular}{|c|c|c|c|c|c|}
\hline & & All & TBI & nTBI & $\begin{array}{c}\text { TBI vs nTBI } \\
p \text {-value } \\
\end{array}$ \\
\hline \multirow[t]{3}{*}{ CAP } & Number of symptoms & $\begin{array}{c}4[3,5] \\
n=30\end{array}$ & $\begin{array}{c}4[3,5] \\
n=18\end{array}$ & $\begin{array}{c}5[3.5,5] \\
n=12\end{array}$ & 0.48 \\
\hline & $\begin{array}{l}\text { Cognitive impairment } \\
\text { subscale score }\end{array}$ & $\begin{array}{c}8[4,10.5] \\
n=40\end{array}$ & $\begin{array}{c}8[4,11] \\
n=26\end{array}$ & $\begin{array}{c}7[4,10] \\
n=14\end{array}$ & 0.64 \\
\hline & GOAT total score & $\begin{array}{c}40.5[20,52] \\
n=42\end{array}$ & $\begin{array}{c}35.5[21,52] \\
n=26\end{array}$ & $\begin{array}{c}44.5[13,52] \\
n=16\end{array}$ & 0.88 \\
\hline DRS & Total score & $\begin{array}{c}14.5[13,16] \\
n=140\end{array}$ & $\begin{array}{c}15[13,16] \\
n=84\end{array}$ & $\begin{array}{c}14[13,16] \\
n=56\end{array}$ & 0.15 \\
\hline
\end{tabular}

All summary values are presented as median [interquartile range] with differences between TBI (traumatic brain injury) and nTBI (non-traumatic brain injury) calculated with a Mann-Whitney U Test. The CAP (Confusion Assessment Protocol) was completed within 7 days of transition from VS (vegetative state) to MCS (minimally conscious state). The DRS (Disability Rating Scale) was completed within 3 days of transition from VS to MCS. The number of CAP symptoms is reported only for those subjects who had a valid assessment of all items on the CAP. Cognitive impairment and GOAT (Galveston Orientation and Amnesia Test) scores are reported for those subjects with valid assessments on each of these measures. 


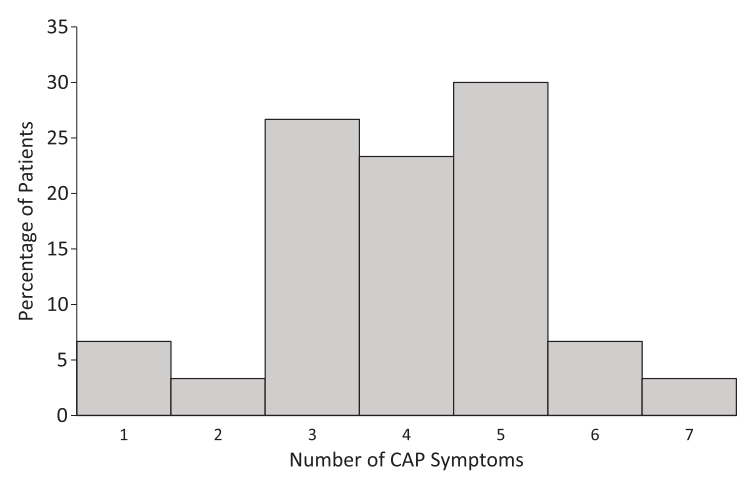

Fig. 2. Distribution of number of CAP symptoms present at time of eMCS.

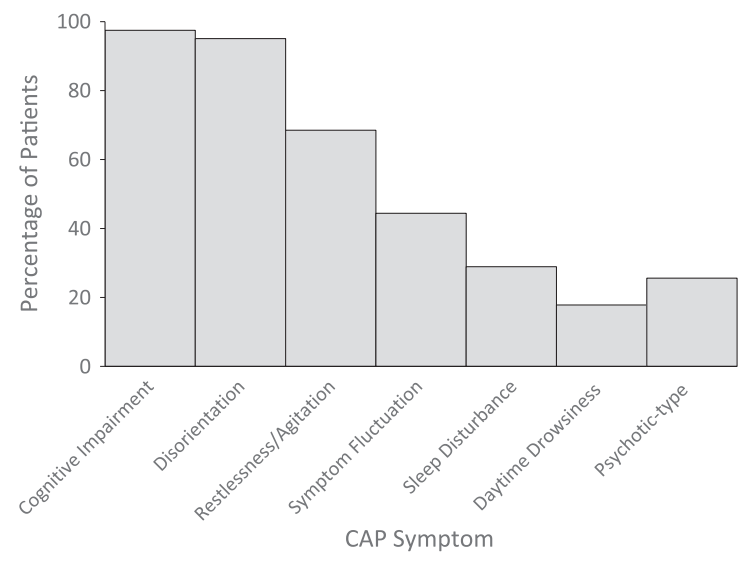

Fig. 3. Distribution of CAP symptom categories present at time of eMCS.

demonstrate characteristics of ACS, including severe cognitive impairment, disorientation, agitation, and symptom fluctuation. Our findings are consistent with studies demonstrating that confusion is associated with both disorientation and impairment across multiple cognitive domains (Sherer et al., 2005; Stuss et al., 1999; Wilson, Baddeley, Shiel, \& Patton, 1992). One notable distinction is that we observed overall lower rates of clinical symptoms on the CAP (Sherer et al., 2005; Sherer et al., 2008; Sherer et al., 2013). Whereas previous studies included all patients at admission to rehabilitation, regardless of how long they were in a confusional state, we focused on capturing the transition from MCS to ACS. During this phase of recovery, patients demonstrate a more restricted range of behavioral output that may prevents expression of some behaviors such as psychotic-type symptoms. Alternatively, variability may exist across settings in how clinicians rate the CAP clinical symptoms, as the clinical

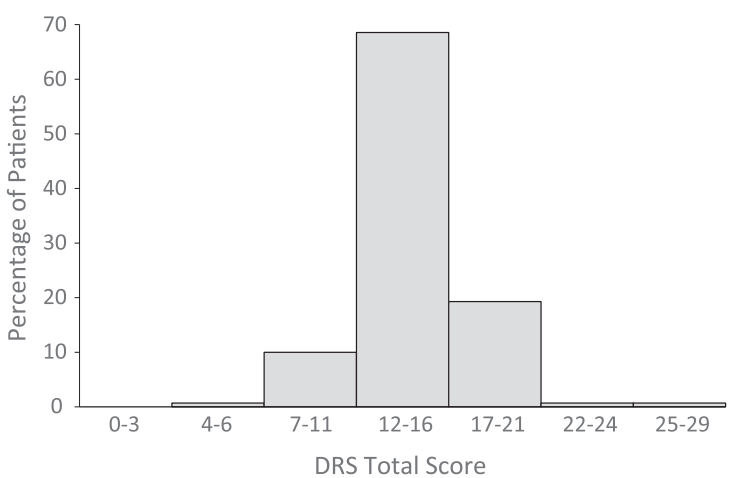

Fig. 4. Distribution of DRS total scores at eMCS. Level of Disability Key: $0=$ None, $1=$ Mild, $2-3=$ Partial, 4-6=Moderate; $7-11=$ Moderately severe; $12-16=$ Severe; 22-24=Vegetative state; 25-29=Extreme vegetative state (http://www.tbims.org/combi/drs).

symptoms are based on observation rather than performance.

Functionally, eMCS is associated with severe disability on the DRS, with patients remaining cognitively and physically dependent on caregivers for completion of basic daily activities. Patients with traumatic and non-traumatic DoC demonstrate similar cognitive and functional profiles upon emergence from MCS, suggesting that assessments and treatments developed for patients in PTCS may be extended to patients with nTBI, and vice versa.

It has been nearly 100 years since Symonds (Symonds, 1937) and Russell (Russell, 1932) published their seminal findings on "clouded consciousness" after TBI. Yet, the phenomenology, functional implications, and pathophysiology of ACS remain largely unstudied. This is, in part, because the term post-traumatic amnesia (PTA) was quickly adopted to describe the period of recovery that follows recovery of consciousness after TBI, unintentionally focusing both investigators and clinicians on the amnestic component of the syndrome rather than the multidimensional characteristics of ACS. However, in 1999, it was suggested that the term PTCS replace PTA because patients in PTA demonstrate a complex combination of cognitive impairments alongside disorientation and memory disturbance (Stuss et al., 1999). Although the state that follows MCS is increasingly seen as having multiple impairments of cognition and behavior, a case definition has not been published and the prognostic implications of ACS remain largely unknown.

When accounting for differences in age and sex, eMCS diagnosis occurred earlier in the recovery 
course for subjects with TBI versus nTBI, but, etiology did not affect degree of confusion (i.e., number of CAP symptoms) or disability at eMCS. Prior investigations have suggested that patients with nTBI have a slower recovery rate and poorer prognosis than those with TBI (Giacino \& Kalmar, 1997). Although our results support a slower recovery rate for nTBI, we show that etiology does not affect cognition, behavior, or function once eMCS is reached. Thus, for patients who are on an upward trajectory of recovery from DoC, etiology may not affect level of function long-term. Alternatively, the CAP may be insensitive to detecting differences in patients with TBI versus nTBI or differences may become more pronounced further in the recovery process. More work is needed to validate the CAP in patients with nTBI and to determine whether the confusional states following TBI and nTBI are similar phenomena, as suggested by a theory that a unified neurochemical process forms the basis for confusion, (Silver, McAllister, \& Yudofsky, 2011) or whether there are underlying differences that our methods could not detect.

Patients emerging from MCS did not score at the floor of either the cognitive impairment or the disorientation items of CAP. This suggests that patients emerging from MCS can correctly respond to at least some items assessing cognition and orientation. Although our dataset did not allow us to investigate whether patients still in MCS could respond to these items, it is possible that some patients who have not yet emerged from MCS may demonstrate signs of confusion. If this is the case, investigators may need to re-examine the upper boundary of MCS and the lower boundary of ACS and consider modifying current behavioral criteria to better establish the border between these two clinical states (Nakase-Richardson et al., 2008). Furthermore, given our empirical evidence that nearly all patients upon emergence from MCS were acutely confused, the terms eMCS and ACS seem to describe a converging stage of recovery. This supports a 2014 review which describes patients newly emerged from MCS as being acutely confused (Giacino et al., 2014). A larger, prospective study with an independent sample will be needed to confirm that eMCS and ACS are fully interchangeable.

We observed a large spread in DRS total scores, suggesting patients range from moderate disability to VS at eMCS. A DRS rating of VS contradicts our criteria that all subjects are eMCS based on CRS$\mathrm{R}$ assessment. Both subjects who demonstrated DRS scores in the VS range were assessed on the DRS three days before the first CRS-R score indicated eMCS.
Thus, it is possible that the discrepancy in DRS and CRS-R scores reflects a rapid improvement in level of function that would have been less pronounced if the DRS were assessed on the day of the first CRS-R exam indicating eMCS. Critically, unlike the CRS-R, the DRS is not designed to detect levels of consciousness, and the CRS-R has been shown to have a higher sensitivity for detecting consciousness than the DRS (Giacino et al., 2004).

Characterizing cognitive and functional disability at eMCS has implications for clinicians, caregivers and researchers. For clinicians, anticipating the marked inattention, memory disturbance, disorientation and overarching behavioral dysregulation that dominate ACS may aid in planning treatment and establishing rehabilitation goals. For families and caregivers, witnessing the return of consistent responsiveness and basic functional communication in the context of confusion, poor awareness and dysregulated behaviors can be distressing. Families should be educated that eMCS is accompanied by ACS and ongoing functional impairment, rather than return of independence. For investigators studying severe brain injury, it is important to understand that MCS is followed by an ongoing state of impaired consciousness, or "clouded consciousness" that should be considered part of the spectrum of DoC. Given that eMCS patients can participate in basic assessment of cognition and orientation, investigators may consider incorporating standardized assessment of ACS into research protocols.

In this retrospective database study, we analyzed clinical metrics collected through a single inpatient specialized DoC program. Consequently, despite using standardized metrics, local variability in administration of the measures may contribute to the findings. Complete and valid CAP assessments within seven days of emergence from MCS were obtained in less than 30 patients and we were unable to identify the reason most patients were missing CAP data. Given that there were no differences in clinical characteristics (e.g., time from injury to emergence from MCS) between the group with and without CAP data, it is possible that the missing data is a result of treating team decision-making. A more robust sampling strategy that documents factors underlying missing data is required to improve the generalizability of our findings. A subset of subjects who attempted the CAP could not complete all aspects of the CAP for various injury-related factors. For example, in subjects with aphasia, only the clinician-reported observational items of the CAP (e.g., symptom fluc- 
tuation, sleep disturbance) could be rated validly. Conversely, performance-based items (i.e., cognitive impairment and GOAT) were occasionally rated in the absence of clinician-reported observational items. These discrepancies highlight the need for designing measures that are sensitive to level of function across the spectrum of recovery and training clinicians on early use of these measures. It is also critical that confounding factors are recorded using a standardized system so that invalid scores are not misinterpreted to be low or missing scores. Systematic use of test completion codes to address factors contributing to missing or invalid scores will be useful for future studies. The median CAP score in this study was obtained four days after eMCS. Early and frequent assessment of confusion may lead to alternate findings.

\section{Conclusion}

eMCS patients demonstrate ongoing, marked disability and significant impairments in attention, memory, and orientation consistent with ACS. Furthermore, as the repertoire of behavioral output increases, there may be restlessness, delusions or hallucinations, and disrupted sleep/wake cycles. These symptoms may wax and wane over minutes, hours, or days. Characterizing the cognitive and functional impairments present at the transition between MCS and ACS is critical for understanding the trajectory of recovery from severe TBI, planning treatment, communicating expectations regarding stages of recovery to families, and conducting research focused on the natural history of recovery from coma.

\section{Acknowledgments}

We wish to thank the therapists and nurses who have embraced the mission of the specialized DoC program and diligently collect the clinical metrics that make this research possible.

\section{Funding sources}

Y. Bodien: National Institute on Disability, Independent Living and Rehabilitation Research (NIDILRR), Administration for Community Living (NIDILRR grant number 90DP0039), James S. McDonnell Foundation.
J. Ostrow: None.

K. Sheau: None.

G. Martens: University and University Hospital of Liège, the Belgian National Funds for Scientific Research (FRS-FNRS), European Commission: Luminous project (EU-H2020-fetopen-ga686764); Human Brain Project (EU-H2020-fetflagship-hbpsga1-ga720270); DOCMA project (EU-H2020MSCA-RISE-778234), IAP research network P7/06 of the Belgian Government (Belgian Science Policy), Wallonie-Bruxelles International (WBI.World), Wallonia-Brussels Federation Travel Grant.

J. Giacino: National Institute on Disability, Independent Living, and Rehabilitation Research (NIDILRR grant number 90DP0039), James S. McDonnell Foundation, National Institutes of Health, U.S. Department of Defense, the National Institute of Neurological Disorders and Stroke.

\section{Conflict of interest}

Y. Bodien is a member of the American Congress of Rehabilitation Medicine (ACRM), the Brain Injury Special Interest Group, and the Disorders of Consciousness Task Force.

$\mathrm{J}$. Giacino is a member of the American Congress of Rehabilitation Medicine (ACRM), the Brain Injury Special Interest Group, and the Disorders of Consciousness Task Force; serves on a scientific advisory board for TBI Model Systems National Data and Statistical Center; has received funding for travel from the US Department of Defense for a meeting related to the TBI Endpoint Development Project, the National Institute on Neurological Disorders and Stroke for the Traumatic Brain Injury Model Systems Project Directors meeting, and for a meeting related to the Transforming Research and Clinical Knowledge in Traumatic Brain Injury study, the National Institute on Disability, Independent Living and Rehabilitation Research, the American Academy of Physical Medicine and Rehabilitation, the One Mind Foundation, the James S. McDonnell Foundation for a meeting related to the Recovery of Consciousness After Severe Brain Injury study, the Barbara Epstein Foundation, and the International Brain Injury Association; has received a cash donation from the Epstein Foundation for a hospital clinical program that he directs and for serving on a team that provided clinical consultation services to an overseas patient who sustained severe brain injury; has served as an editor for the Journal of Head Trauma Rehabilitation; 
has received honoraria from the One Mind Foundation, Holy Cross Hospital (Surrey, UK); HealthSouth Braintree Hospital, Western Michigan Brain Injury Network, George Washington University Medical School, Association of Academic Physiatrists, Mayo Clinic, Kennedy-Krieger Institute, and Magill's Medical Guide; performs clinical procedures as $10 \%$ of his clinical effort in his role as Director of Spaulding Rehabilitation Network Disorders of Consciousness Program and neuroimaging as a PI on 2 neuroimaging studies for $30 \%$ of his research effort; received financial support from the NIH-National Institute on Neurological Disorders and Stroke (NINDS) for Central Thalamic Stimulation for Traumatic Brain Injury, U. S. Department of Defense for TBI Endpoint Development Project, the Huperzine A for the Treatment of Cognitive, Mood and Functional Deficits After Moderate and Severe TBI study, the INjury and TRaumatic STress (INTRuST) Consortium Neuroimaging Acquisition and Archival study, the National Institute on Disability, Independent Living and Rehabilitation Research (NIDILRR) for the Spaulding Harvard - Traumatic Brain Injury Model System and for Multicenter Evaluation of Memory Remediation after traumatic Brain Injury with Donepezil, National Institute for Neurologic Disorders and Stroke (NINDS) for Transforming Research and Clinical Knowledge in Traumatic Brain Injury study, James S. McDonnell Foundation for Study of Recovery of Consciousness After Severe Brain Injury, Barbara Epstein Foundation, and the Spaulding Rehabilitation Hospital Department of Physical Medicine and Rehabilitation; and has acted as a witness with regard to a legal proceeding.

\section{References}

Bogner, J. A., Corrigan, J. D., Stange, M., \& Rabold, D. (1999). Reliability of the Agitated Behavior Scale. J Head Trauma Rehabil, 14(1), 91-96.

Giacino, J. T., Ashwal, S., Childs, N., Cranford, R., Jennett, B., Katz, D. I.,... Zasler, N. D. (2002). The minimally conscious state: Definition and diagnostic criteria. Neurology, 58(3), 349-353.

Giacino, J. T. (2004). The vegetative and minimally conscious states: Consensus-based criteria for establishing diagnosis and prognosis. NeuroRehabilitation, 19(4), 293-298.

Giacino, J. T., Fins, J. J., Laureys, S., \& Schiff, N. D. (2014). Disorders of consciousness after acquired brain injury: The state of the science. Nat Rev Neurol, 10(2), 99-114. doi:10.1038/nrneurol.2013.279

Giacino, J. T., \& Kalmar, K. (1997). The vegetative and minimally conscious states: A comparison of clinical features and functional outcome. J Head Trauma Rehabil, 12(4), 36-51.

Giacino, J. T., Kalmar, K., \& Whyte, J. (2004). The JFK Coma Recovery Scale-Revised: Measurement characteristics and diagnostic utility. Arch Phys Med Rehabil, 85(12), 2020-2029.

Harris, P. A., Taylor, R., Thielke, R., Payne, J., Gonzalez, N., \& Conde, J. G. (2009). Research electronic data capture (REDCap)-a metadata-driven methodology and workflow process for providing translational research informatics support. $J$ Biomed Inform, 42(2), 377-381. doi:10.1016/j.jbi.2008.08.010

Levin, H. S., O'Donnell, V. M., \& Grossman, R. G. (1979). The Galveston Orientation and Amnesia Test. A practical scale to assess cognition after head injury. J Nerv Ment Dis, 167(11), 675-684.

Nakase-Richardson, R., Yablon, S. A., \& Sherer, M. (2007). Prospective comparison of acute confusion severity with duration of post-traumatic amnesia in predicting employment outcome after traumatic brain injury. J Neurol Neurosurg Psychiatry, 78(8), 872-876. doi:10.1136/jnnp.2006.104190

Nakase-Richardson, R., Yablon, S. A., Sherer, M., Evans, C. C., \& Nick, T. G. (2008). Serial yes/no reliability after traumatic brain injury: Implications regarding the operational criteria for emergence from the minimally conscious state. J Neurol Neurosurg Psychiatry, 79(2), 216-218. doi:10.1136/jnnp.2007.127795

Nakase-Richardson, R., Yablon, S. A., Sherer, M., Nick, T. G., \& Evans, C. C. (2009). Emergence from minimally conscious state: Insights from evaluation of posttraumatic confusion. Neurology, 73(14), 1120-1126. doi:10.1212/WNL.0b013e3181bacf34

Nakase-Thompson, R., Sherer, M., Yablon, S. A., Nick, T. G., \& Trzepacz, P. T. (2004). Acute confusion following traumatic brain injury. Brain Injury, 18(2), 131-142.

Rappaport, M., Hall, K. M., Hopkins, K., Belleza, T., \& Cope, D. N. (1982). Disability rating scale for severe head trauma: Coma to community. Arch Phys Med Rehabil, 63(3), 118-123.

Russell, W. R. (1932). Brain Involvement in Head Injury. A Preliminary Study of 200 Cases. Edinb Med J, 39(2), T25-T42.

Sherer, M., Nakase-Thompson, R., Yablon, S. A., \& Gontkovsky, S. T. (2005). Multidimensional assessment of acute confusion after traumatic brain injury. Archives of Physical Medicine \& Rehabilitation, 86(5), 896-904.

Sherer, M., Yablon, S. A., Nakase-Richardson, R., \& Nick, T. G. (2008). Effect of severity of post-traumatic confusion and its constituent symptoms on outcome after traumatic brain injury. Arch Phys Med Rehabil, 89(1), 42-47. doi:10.1016/j.apmr.2007.08.128

Sherer, M., Yablon, S. A., \& Nick, T. G. (2013). Psychotic symptoms as manifestations of the posttraumatic confusional state: Prevalence, risk factors, and association with outcome. Journal of Head Trauma Rehabilitation.

Silva, M. A., Nakase-Richardson, R., Sherer, M., Barnett, S. D., Evans, C. C., \& Yablon, S. A. (2012). Posttraumatic confusion predicts patient cooperation during traumatic brain injury rehabilitation. Am J Phys Med Rehabil, 91(10), 890-893. doi:10.1097/PHM.0b013e31825a1648

Silver, J., McAllister, T., \& Yudofsky, S. (2011). Textbook of Traumatic Brain Injury. In (2 ed.). Washington, DC: American Psychiatric Pub.

StataCorp. (2015). Stata Statistical Software: Release 14. In. College Station, TX: StataCorp LP.

Stuss, D. T., Binns, M. A., Carruth, F. G., Levine, B., Brandys, C. E., Moulton, R. J.,... Schwartz, M. L. (1999). The acute period 
of recovery from traumatic brain injury: Posttraumatic amnesia or posttraumatic confusional state? J Neurosurg, 90(4), 635643.

Symonds, C. P. (1937). Mental Disorder Following Head Injury: (Section of Psychiatry). Proc R Soc Med, 30(9), 1081-1094.

Trzepacz, P. T., Mittal, D., Torres, R., Kanary, K., Norton, J., \& Jimerson, N. (2001). Validation of the Delirium Rating Scalerevised-98: Comparison with the delirium rating scale and the cognitive test for delirium. J Neuropsychiatry Clin Neurosci, 13(2), 229-242. doi:10.1176/jnp.13.2.229

Wilson, B. A., Baddeley, A., Shiel, A., \& Patton, G. (1992). How does post-traumatic amnesia differ from the amnesic syndrome and from chronic memory impairment? Neuropsychological Rehabilitation, 2(3), 231-243. doi:10.1080/09602019208401410 